\title{
Le pont d'en haut, le pont d'en bas, et celui qui n'existe pas - Exemple de coopération frontalière à Görlitz et Zgorzelec
}

Upper bridge, lower bridge and bridge which does not exist - Example of cross border cooperation in Görlitz and Zgorzelec

Die obere Brücke, die untere Brücke, die nicht besteht - Beispiel der grenzüberschreitenden Zusammenarbeit in Görlitz und Zgorzelec

\section{Aleksandra Galasinska}

Traducteur : Catherine Hellegouarc'h

\section{(2) OpenEdition Journals}

Édition électronique

URL : http://journals.openedition.org/rge/2236

DOI : $10.4000 /$ rge. 2236

ISSN : 2108-6478

Éditeur

Association des géographes de l'Est

Édition imprimée

Date de publication : 1 septembre 2003

ISSN : 0035-3213

Référence électronique

Aleksandra Galasinska, « Le pont d'en haut, le pont d'en bas, et celui qui n'existe pas - Exemple de coopération frontalière à Görlitz et Zgorzelec », Revue Géographique de l'Est [En ligne], vol. 43 / 4 | 2003, mis en ligne le 20 novembre 2010, consulté le 08 septembre 2020. URL : http:// journals.openedition.org/rge/2236; DOI : https://doi.org/10.4000/rge.2236

Ce document a été généré automatiquement le 8 septembre 2020

Tous droits réservés 


\title{
Le pont d'en haut, le pont d'en bas, et celui qui n'existe pas - Exemple de coopération frontalière à Görlitz et Zgorzelec
}

\author{
Upper bridge, lower bridge and bridge which does not exist - Example of cross \\ border cooperation in Görlitz and Zgorzelec \\ Die obere Brücke, die untere Brücke, die nicht besteht - Beispiel der \\ grenzüberschreitenden Zusammenarbeit in Görlitz und Zgorzelec
}

Aleksandra Galasinska

Traduction : Catherine Hellegouarc'h

A la mémoire de Krzysztof Bialach.

\section{Introduction}

Görlitz et Zgorzelec sont deux villes qui se situent sur la rivière Neisse, Görlitz sur la rive ouest, et donc en République fédérale d'Allemagne, Zgorzelec sur la rive est et, par conséquent, en Pologne (fig. 1). Avant la redéfinition des frontières politiques en 1945, ces deux villes n'en formaient qu'une, la ville allemande de Görlitz. Les bouleversements historiques ont rapproché les représentants de deux nations qui n'ont rien en commun, en dehors de 50 années de communisme. Leur identité nationale, linguistique et locale, tout comme le passé associé à leur région réciproque, les séparent, ainsi que leur appartenance depuis presque dix ans à deux états européens très différents, traversés et divisés par la rivière Neisse, la frontière de Schengen de l'Europe de l'Est. Cependant, les cinq dernières années ont vu ces deux villes prendre part à une autre expérience historique. Le 5 mai 1998, jour de l'Europe, des membres du conseil de la ville polonaise de Zgorzelec ont traversé le pont frontalier (aussi appelé le 
Pont de l'Amitié) sur la Neisse pour rejoindre la ville voisine sur l'autre berge, la ville allemande de Görlitz. Le but de cette visite officielle était une session commune des deux conseils municipaux et la signature de la création de Zgorzelec-Görlitz en tant qu'«Eurocité». Cette déclaration s'accompagna d'un événement artistique où fut inauguré le WAZE (abréviation polonaise d'Image artistique de l'Europe Unifiée), sculpture placée au sommet d'un bâtiment sur la rive droite (polonaise) de la Neisse. Les représentants de Zgorzelec ont emprunté le pont d'en haut (le niveau supérieur du pont, pour traverser la Neisse, accès normalement réservé aux voitures. La circulation frontalière entre l'Allemagne et la Pologne fut donc interrompue un instant. Les piétons traversent habituellement le pont par le niveau inférieur, le pont d'en bas, pour passer plus facilement, mais ce niveau est aussi beaucoup moins impressionnant. C'est ce niveau-là qu'utilisent les habitants de Görlitz et de Zgorzelec tous les jours. Cet article tente de démontrer que, à l'image des deux niveaux du pont, la coopération frontalière des deux communautés s'opère aussi sur deux niveaux (Parker Pearson and Richards, 1994). Ainsi, on peut parler du niveau supérieur officiel, représenté par les élites politiques et du niveau inférieur, celui du quotidien des habitants moyens de Görlitz et de Zgorzelec. Les résultats des recherches entreprises dans les deux villes révèlent un décalage et une absence de communication entre les deux niveaux ou, en d'autres termes, entre le discours des représentants politiques et celui des communautés locales. Comme c'est souvent le cas lors de situations réelles conçues en termes d'opposition, il y a aussi un élément ambivalent de médiation qui pourrait toutefois permettre aux visions de l'Eurocité de prendre forme et cohérence. Cet élément est une autre forme de traverse frontalière, un pont exclusivement réservé à l'usage des piétons, un pont qui relierait le vieux quartier de Görlitz à celui de Zgorzelec. Il est prévu que ce pont soit construit à l'endroit même de l'Altstadtbrücke (le pont de la vieille ville), qui existait au Moyen Age, et qu'il constitue la voie de transport principale entre ce qui était le centre de Görlitz sur la rive gauche et ses quartiers résidentiels sur la rive droite. Mais ce pont piétonnier n'existe pas encore. 
Figure 1 : L'Eurorégion Neisse/Nysa/Nisa.

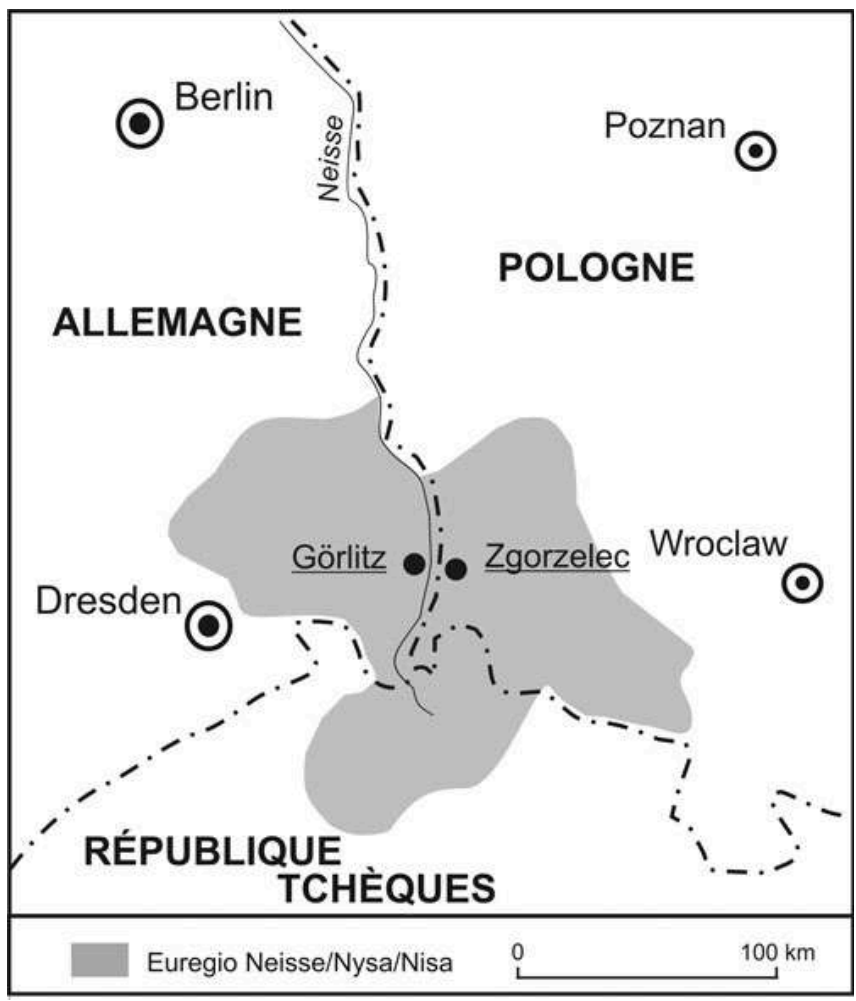

\section{L'histoire de deux villes}

\section{Görlitz avant-guerre}

Avant la Deuxième Guerre mondiale, Görlitz était une grande ville prospère située sur la Via Regia reliant l'Europe de l'ouest à l'Europe de l'est (Francfort sur le Main-DresdeCracovie-Kiev), la voie d'accès à la province allemande de Silésie, qui fut cédée à la Pologne en 1945. De nombreux habitants de Görlitz sont originaires de cette région et l'adhésion de beaucoup d'entre eux à une identité silésienne plutôt qu'à une identité saxonne imposée par les frontières politiques allemandes actuelles se ressent fortement dans la ville. La population de la ville s'élevait à 94645 habitants sur les deux rives en 1934. Après-guerre, en 1949, la population de Görlitz seule (c'est-à-dire de la rive gauche uniquement) comptait 101742 habitants. Cet accroissement était dû à l'arrivée de réfugiés des territoires cédés à la Pologne.

Le textile et la fabrication de wagons ferroviaires étaient les principales industries d'avant-guerre. Les personnes qui nous ont renseignés se souviennent des usines de tannage sur la rive droite au nord de la ville. Des quartiers résidentiels bourgeois s'étendent désormais au sud de la rive droite de Görlitz, avec un grand parc, la salle commémorative du Haut-Laustitz et des casernes militaires. Sur la rive gauche de la Neisse, on trouve la partie la plus ancienne de la ville avec ses deux places de marché et les hautes flèches de l'église Saint-Pierre. En fait, l'église est encore aujourd'hui l'élément le plus caractéristique de la structure urbaine de Görlitz. Des restaurants, des cafés, des locations de canoë-kayaks se répartissaient sur les rives de la Neisse. La rivière était ainsi au cœur des activités de loisir et de divertissement pour les habitants 
de cette ville prospère - ce qui se caractérisait par les éléments typiques des grandes villes (commerce et industrie) et ceux d'une ville d'eau (la proximité de la rivière). Les deux rives de la ville étaient reliées par sept ponts. A la fin de la guerre, les ponts de la Neisse furent détruits par l'armée nazie en retraite. Seul le pont frontalier fut reconstruit après-guerre.

4 La Conférence de Potsdam de 1945 établit la "Ligne Oder-Neisse » comme nouvelle frontière entre la Pologne et l'Allemagne, divisant ainsi toutes les villes situées de part et d'autre de la Neisse. En dehors des petites communautés et des villages, cette division fut ressentie au niveau de grandes villes telles que Francfort sur l'Oder, Guben et Görlitz. Les Alliés ont donc décidé de diviser Görlitz en une ville polonaise, Zgorzelec, à l'est, et une ville allemande, Görlitz, à l'ouest.

\section{Zgorzelec}

5 L'armée soviétique s'empara de la rive droite de Görlitz le 8 mai 1945. Le 10 mai 1945, les autorités polonaises arrivaient dans la ville et organisaient l'administration polonaise de la ville. Le nouveau nom de Zgorzelice fut donné à la partie est de la ville qui, en 1946, comptait 5261 habitants. En 1948, le Comité de la Nomenclature (Komisja Ustalania Nazw Miejscowych) changea le nom de cette nouvelle ville polonaise en faveur de Zgorzelec, plus révélateur des origines slaves du lieu. Presque aussitôt, on a pu distinguer quatre groupes caractéristiques parmi le flux des nouveaux habitants :

1. des Polonais revenant du Front de l'ouest (des soldats et des travailleurs forcés);

2. des prisonniers de guerre libérés du camp Stalag VIII A, installé à Görlitz en 1939 ;

3. des Polonais du centre de la Pologne ;

4. des gens chassés des territoires frontaliers repris par l'Union Soviétique (les régions frontalières de l'est de la Pologne avant-guerre) ;

5. du personnel militaire.

6 À partir d'août 1946, les autorités polonaises commencèrent un rapatriement systématique de la population allemande qui vivait ou qui avait été déplacée dans la ville, ce qui signifie qu'il n'y a plus aucun Allemand aujourd'hui à Zgorzelec. Il est aussi important de noter que les Polonais qui vivent aujourd'hui à Zgorzelec n'ont jamais connu le Görlitz d'avant-guerre. Par conséquent, ceux-là même qui sont aujourd'hui voisins de part et d'autre de la Neisse ne s'étaient jamais rencontrés avant 1945 , n'avaient jamais partagé de région frontalière, et encore moins créé d'identité locale. C'est le déroulement de l'histoire européenne qui a réuni ces populations pour en faire des voisins.

7 À la fin de 1949, 15000 réfugiés politiques grecs et macédoniens furent installés dans la ville. Les chiffres du Bureau des statistiques de Pologne (GUS) les évaluent à 13562 réfugiés en 1950. La plupart d'entre eux se répartirent dans d'autres régions de la Pologne ou finirent par retourner en Grèce dans les années 80 . Actuellement, il ne reste à Zgorzelec qu'environ 200 habitants d'origine grecque.

8 C'est à Zgorzelec que fut signé le 6 juillet 1950 ce qu'on appelle l'«Accord de Zgorzelec », un traité confirmant la frontière séparant la Pologne et la RDA. L'« Accord de Zgorzelec » fut le seul traité entre la Pologne et l'un des deux états allemands jusqu'à la signature du traité frontalier avec la République fédérale d'Allemagne unifiée en 1991. Ce traité marque le point final à des années de doute quant au statut de la ligne 
Oder-Neisse comme frontière reconnue par le gouvernement de Bonn. L'ouverture de la frontière entre les deux villes ne fut autorisée qu'en 1971. La frontière fut fermée à nouveau en 1981, lors de l'introduction de la loi martiale en Pologne, et réouverte durant le processus de démocratisation en Pologne à la fin des années 80 .

Après la guerre, l'essor économique de la ville repose sur l'ouverture de mines de lignite alimentant une centrale électrique construite près de la ville entre 1959 et 1965. Les mines et la centrale sont donc devenues les sources principales d'emploi pour la population de Zgorzelec et expliquent le taux relativement bas de chômage en comparaison des autres régions frontalières de l'ouest de la Pologne (autour de $14 \%$ en 2000). Ce développement industriel marqua aussi le commencement de l'investissement polonais dans la ville, particulièrement dans les domaines du logement, des équipements sportifs et du commerce. En 1960, Zgorzelec comptait 16037 habitants alors que dix ans plus tard, en 1970, il y en avait 28 472. Cette tendance à la croissance se maintint jusqu'en 1990 (36103 habitants). On a pu constater une légère baisse depuis (2000: 35690 habitants ; 2001: 35473 habitants). Zgorzelec se situe dans la province (en polonais : wojewodztwo) de la Basse-Silésie, et constitue le chef-lieu de la région (en polonais, powiat).

\section{Görlitz après-guerre}

Le nouveau tracé de la frontière après la guerre laissa le centre-ville historique, la gare principale et la majorité des bâtiments de la ville (peu endommagés par la guerre) en territoire allemand. Il n'en resta pas moins un sentiment énorme de perte et d'amputation. En 1948, un procès pour crimes de guerre, tenu dans la salle du Stadthalle près de la Neisse, condamna à mort l'ancien maire de la ville sous les Nazis, Hans Meinshausen, ainsi que le chef du parti nazi local. La révolte du 17 juin 1953 contre le gouvernement de la RDA fut particulièrement intense à Görlitz. La population s'empara de l'Hôtel de ville et libéra des prisonniers politiques.

11 La rivière, jadis symbole de loisir et de récréation pour la ville, perdit son rôle. Seuls le viaduc ferroviaire et l'ancien pont Reichenberger (renommé le Pont de l'Amitié en 1951) furent reconstruits. La frontière était sous contrôle très strict et interdite au public jusqu'en 1971. Les relations frontalières étaient minimales et n'impliquaient guère que des ouvriers polonais travaillant dans les usines de Görlitz, comme l'usine Condenser sur les bords de la Neisse, clairement visible à partir des deux villes. Aujourd'hui, cette usine est désaffectée, symbole du déclin économique des deux villes. Seules l'industrie du textile et la construction ferroviaire se sont maintenues.

12 Le rôle historique de la ville en tant que porte d'accès à la Silésie et ville importante sur la Via Regia entre l'Europe de l'Ouest et de l'Est a disparu. L'ouverture de la frontière en 1971 constitua la première occasion de visiter Zgorzelec ainsi que les régions montagneuses de la Basse-Silésie, jadis site touristique allemand prisé et pays d'origine de nombreux citoyens de Görlitz. Cependant, l'état est-allemand ferma à nouveau la frontière en 1981, craignant que le courant révolutionnaire grandissant de Solidarnosc ne se répande au delà de la Neisse.

13 Une grande partie de la population de Görlitz adhéra à la vague de protestations qui s'éleva contre le gouvernement est-allemand vers la fin de l'année 1989. Début novembre, plus de 5000 personnes assistaient aux «Prières pour la Paix » dans les 
églises protestantes de la ville. En l'espace de quelques semaines, Görlitz se trouvait dans un état qui négociait son absorption à l'intérieur d'une Allemagne réunifiée.

Lorsqu'elle faisait partie de la RDA, la ville de Görlitz se situait dans la région («Bezirk») de Dresde. Dans la République fédérale unifiée, la ville se trouve dans le Land de Saxe. En 1985 la population était tombée à 79277 habitants, cette baisse se confirma après la réunification $(1990: 72237 ; 2001: 60600)$. Depuis 1990 la ville a donc perdu près de $20 \%$ de sa population. Le taux de chômage en avril 2000 atteignait $21,7 \%$, soit 8614 chômeurs, dont 49,3\% d'hommes, une proportion anormalement élevée pour la région.

\section{Une histoire commune depuis 1991}

Depuis mai 1991, Görlitz et Zgorzelec font partie de l'Eurorégion de la Neisse, qui comprend l'est de la Saxe en Allemagne, la Basse-Silésie en Pologne et le nord de la République tchèque (fig. 1). Les villes ont signé un accord de coopération en 1991. En 1996, la première session commune entre les conseils de Zgorzelec et de Görlitz fut tenue le jour où il fut déclaré par les deux villes qu'elles allaient commémorer le neufcent-vingt-cinquième anniversaire de la fondation de la ville. Depuis 1998, la session commune des deux conseils est un événement annuel.

Il y a trois postes frontière dans la ville et ses environs immédiats. Un des postes se situe dans la ville elle-même, un autre sur l'autoroute ouverte depuis 1996 (Jedrzychowice-Ludwigsdorf) et un autre encore sur l'ancienne voie de chemin de fer allemande de 1846 (fig. 1). L'ouverture réelle de la frontière a eu lieu en 1989, il suffit donc désormais aux riverains de montrer leur carte d'identité pour la traverser (Jajesniak-Quast \& Stoklosa, 2000 ; Kennard A., 2000 ; Szlachcicowa, 1999 ; Osekowski et Szczegola, 1999).

\section{La vue du pont d'en haut}

On peut remarquer la coopération exemplaire des partenaires lorsque l'on s'adresse aux autorités des deux communautés, que l'on consulte les sites internet officiels de Görlitz et de Zgorzelec, la presse locale ou nationale, ou encore si l'on observe le développement du programme "Stadt 2030 ». Les deux villes, ou la cité unique européenne, coopèrent sur quatre points particuliers: a) l'urbanisation; b) la protection de l'environnement ; c) la culture, le sport et l'éducation ; d) la justice. Cette coopération a donné lieu à deux initiatives concernant les transports : une ligne de bus régulière qui traverse la frontière et relie le centre de Görlitz à un grand supermarché de Zgorzelec ainsi que la libre circulation des taxis de part et d'autre de la frontière. Les villes espèrent aussi construire une ligne de tramway commune qui serait en fait une restauration de la ligne de tramway d'avant-guerre qui traversait la rivière et elles ont lancé un programme de restauration architecturale des bâtiments d'avant-guerre sur la rive polonaise. Il est aussi prévu de reconstruire les anciens ponts piétonniers. Görlitz a ouvert un office de la culture et de la communication à Zgorzelec, qui fait la publicité des événements culturels auprès des Polonais et leur offre des promotions intéressantes sur l'achat des billets. Un hebdomadaire gratuit germano-polonais (Wochenkurier) est distribué à Görlitz et Zgorzelec depuis 1997. Un théâtre de Jelenia Gora monte un spectacle une fois par mois à Görlitz, une association germano- 
polonaise pour les jeunes se trouve à Zgorzelec au Dom Kultry (Maison de la Culture). Cette association s'occupe en particulier des jeunes « difficiles ", à savoir des jeunes qui ont déjà connu des déboires avec la justice. Les activités de réinsertion sociale qui y sont organisées sont gérées par des assistants polonais et allemands. Il y a deux écoles maternelles, une sur chaque rive, ouvertes chacune aux enfants allemands et polonais. Les élèves de Zgorzelec apprennent l'allemand comme deuxième langue et il y a aussi un lycée à Görlitz qui propose le polonais comme deuxième langue.

Interrogés sur la coopération et les problèmes que représente l'Eurocité, les parlementaires mentionnent plutôt la Commission européenne de Bruxelles et les gouvernements nationaux comme sources de difficulté, en particulier en ce qui concerne l'élargissement de l'Union européenne dans les communautés frontalières. Chacun des maires a souligné l'importance de fortes structures bilatérales au niveau de la ville bien avant cet élargissement et a proposé que l'Eurocité de Görlitz-Zgorzelec (Europastadt) soit un modèle à suivre pour les Eurocités futures, avec sa structure politique et son comité, ainsi que sa stratégie de marketing communs. Ces structures établies peuvent en effet se maintenir au-delà des changements politiques, d'autant qu'elles encouragent les représentants des administrations des deux villes à travailler ensemble. Ce travail est rendu particulièrement difficile par les différentes démarches de part et d'autre de la frontière ; Görlitz, par exemple, doit s'adresser au parlement du Land qui épluche et adapte les réformes avant de les présenter à Bruxelles, alors que Zgorzelec doit s'adresser à Varsovie. La distance de Varsovie qui est située à près de 500 $\mathrm{km}$, complique les relations avec une petite ville éloignée dans le sud ouest du pays et ralentit le processus de décision. Le nouveau INTERREG III fut pressenti comme solution possible à ces problèmes, beaucoup de personnes souhaitant traiter directement avec Bruxelles. Les propositions de projets ne peuvent être révisées ou améliorées car l'Union européenne n'explique pas ses rejets. L'incertitude et les retards des subventions exercent une pression énorme sur les budgets régionaux et n'encouragent guère les propositions futures, surtout quand des subventions égales sont nécessaires à la réalisation des projets.

Mais cette vision d'une coopération harmonieuse dont les seules difficultés ne proviendraient que de l'extérieur ne semble vraie que si l'on traverse le pont d'en haut. Cette vision privilégiée ne peut en effet s'appliquer que pour ceux qui n'envisagent pas l'arrêt du trafic frontalier international comme un problème. Cela dit, un jour qu'il revenait d'un rassemblement officiel de jeunes de Görlitz et de Zgorzelec, le maire d'une des deux villes dû faire la queue comme tout le monde et attendre son tour pour pouvoir traverser la rivière, malgré la signalisation de son véhicule et ses explications disant qu'il était en voyage officiel et non en sortie shopping sur l'autre rive. Cet incident mit en évidence le décalage entre la réalité internationale des accords de Schengen et celle des Eurocités. Il est possible cependant que ceux qui n'empruntent que le pont d'en bas aient ressenti quelque satisfaction à voir les autorités faire la queue. Cependant, ils pouvaient être frustrés par la fiction de la propagande de coopération face à la brutalité du quotidien.

\section{La vue du pont d'en bas}

Nous avons pu acquérir un statut d'observateurs actifs de la vie frontalière après avoir vécu au sein des communautés pendant plusieurs mois. Nous nous sommes même 
fondus dans ces communautés. Nous avons traversé le « Pont de l'Amitié » presque tous les jours, observant le bas de l'Eurocité qui accueille 99 pour cent des habitants de la communauté. Notre vision de cette Eurocité est celle de deux villes, Görlitz en Allemagne et Zgorzelec en Pologne.

Contrairement à la politique de coopération entre les deux villes, les rapports quotidiens semblent très rares, malgré tous les efforts des organisations culturelles des deux côtés de la rivière (un cas similaire est exposé dans Rabinowitz, 1994 ; Donnan \& Wilson, 1994 ; Donnan \& Wilson, 1999). Les deux communautés vivent l'une à côté de l'autre sans s'intéresser à la vie des uns et des autres, même plutôt en s'en moquant, comme le démontrent nos entretiens avec les habitants. Le shopping est la seule activité que ces deux communautés ont en commun. Les Polonais privilégient Görlitz pour l'achat de vêtements, surtout à la période des soldes, où on les accueille volontiers comme de très bons clients. Les personnes interrogées ont insisté sur le fait qu'il leur est possible de payer avec de la monnaie polonaise à Görlitz. De nombreux magasins emploient d'ailleurs des vendeurs polonais pour permettre à leurs clients de Zgorzelec de communiquer dans leur langue. La langue reste un handicap majeur dans les relations entre les communautés, bien que le polonais s'entende régulièrement à Görlitz, surtout dans les quartiers commerçants.

Nos informateurs ont très souvent souligné un net changement dans l'attitude des commerçants allemands vis-à-vis des clients polonais. Les premières années qui ont suivi l'ouverture de la frontière en 1989 laissent de mauvais souvenirs. Les témoins polonais parlent de situations conflictuelles, de l'impolitesse des commerçants et de leur étroite surveillance des clients polonais pendant toute la durée de leur visite dans les magasins. On peut noter plusieurs causes à cette attitude négative envers les clients polonais. Tout d'abord, c'est le résultat de quarante années de frontière fermée. Les deux nations n'entretenaient pratiquement aucun contact malgré les quelques mètres qui les séparaient. Lorsque l'on se tient sur une rive, on peut non seulement très bien voir les habitants de l'autre rive, mais on peut aussi jeter des objets de l'autre côté de la rivière. Notre enquête enregistre d'ailleurs des prises de contact de ce genre dans les années 50 et 60 où les jeunes échangeaient des chewing-gums, des bonbons, des cigarettes ou des briquets. Ils échangeaient aussi leur adresse et tentaient de créer des liens que l'âge adulte et la fermeture de la frontière rendaient vains (pour une étude similaire, voir aussi Meinhof \& Galasinski, 2000 ; Meinhof \& Galasinski, 2002 ; Galasinski \& Meinhof, 2002).

L'héritage de la Seconde Guerre mondiale est un autre facteur, avec le problème de l'éviction des Allemands des territoires conquis par la propagande communiste polonaise et les "Territoires reconquis» (Ziemie Odzyskane). La fermeture de la frontière et l'absence de bons traités ne fit qu'aggraver l'hostilité entre les deux nations, et ce malgré les campagnes de propagande des deux pays pour l'amitié et la coopération. Il est vrai cependant que ces campagnes se limitaient aux embrassades convenues et accolades chaleureuses entre Erich Honecker et Edward Gierek, secrétaires généraux respectifs des partis communistes est-allemand et polonais dans les années 70. Les jeunes de Görlitz et de Zgorzelec se rencontraient essentiellement au milieu du pont pour brandir des drapeaux.

La situation socio-économique est un troisième facteur d'hostilité entre les deux voisins. Les salaires des gérants de magasins est-allemands ne dépendaient guère des résultats des ventes et les commerçants pouvaient donc se permettre de maltraiter 
leurs clients polonais. Mais les années 90 et la réunification de l'Allemagne ont entrâné la hausse rapide du chômage dans les régions de l'est. Bientôt, l'argent avait remplacé la nationalité comme argument de taille dans les magasins; ceux qui avaient eu un emploi garanti jusqu'à présent n'étaient plus favorablement accueillis. Pour les commerçants de Görlitz, la découverte de nouveaux clients qui traversaient le pont pour venir faire leurs courses signifiait dans certains cas la possibilité de doubler leurs profits. L'arrêt de la soi-disant « juma " peut aussi expliquer ce changement d'attitude (Baczynska, 1999; Kurzepa, 1999): les incessants vols à l'étalage de la jeunesse polonaise. Comme l'offre et la situation économique se sont uniformisées de part et d'autre de la frontière, ces vols ont disparu. Nos informateurs polonais s'accordent tous pour dire que les temps où on les recevait avec méfiance et mépris à Görlitz sont révolus. Ils sont aussi nombreux à trouver que Görlitz est une zone commerçante bien plus attrayante que Zgorzelec, la ligne de bus qui relie les deux villes rend d'ailleurs le shopping très facile.

Par contre, la présence allemande à Zgorzelec est limitée. Nos témoins de Görlitz traversent toutefois aussi la frontière pour se rendre à Zgorzelec, surtout pour le Supermarché Real, les stations d'essence et les coiffeurs. Cependant, le discours polonais de conquête du territoire de Görlitz est remplacé par une certaine prudence et l'évocation de distance et de différences. Zgorzelec, ou du moins la partie au-delà de la zone frontalière immédiate avec ses marchands de tabac, semble bien plus lointaine pour les habitants de Görlitz que Görlitz pour ses voisins polonais. On n'entend plus parler « d'aller au bout de la rue " pour faire des courses comme à Zgorzelec, mais on a plutôt l'impression qu'il s'agit d'un endroit étrange et étranger, presque dangereux où tout peut arriver.

Le point de vue de la rive polonaise offre le panorama magnifique d'une belle ville. La ville de Görlitz est particulièrement belle, la Seconde Guerre mondiale ayant laissé peu de traces sur son architecture. Les dégradations des années d'abandon de la RDA s'estompent aussi peu à peu. Le centre de la ville est restauré intégralement rue par rue, ce qui transforme Görlitz en un endroit agréable pour vivre et travailler et peut même en faire une destination touristique. On peut effectivement remarquer de plus en plus de groupes de Tour Operators et de visites guidées à travers la ville, ce qui est un signe encourageant pour l'économie, au moins pour ce qui est du domaine touristique.

Il semblerait que les habitants de Zgorzelec ne puissent se laisser submerger par la beauté architecturale de leur ville de la même manière. Presque tous les habitants de Zgorzelec interrogés regrettent l'absence de certains espaces, pourtant banals pour d'autres villes ou communautés, ce qui leur laisse l'impression d'une ville incomplète, «presque une ville mais pas tout à fait ». C'est une impression partagée par toutes les générations d'habitants et, de fait, Zgorzelec fut élaborée à partir de quartiers et de banlieues (Galasinska et al., 2002).

Du point de vue culturel, les rencontres sont pour ainsi dire inexistantes. Les Polonais ne se rendent pas à Görlitz pour assister à des événements culturels, ni pour visiter les cafés et restaurants allemands. Les grandes différences de prix sont évoquées ainsi que la proximité de la République tchèque, à seulement $12 \mathrm{~km}$. De nombreux entretiens révèlent aussi la peur irraisonnée de situations potentiellement conflictuelles lorsque des Allemands sont présents. Même si les témoignages directs de telles situations manquent, cette peur irrationnelle demeure. Il ne semble même pas possible ou 
imaginable que les Polonais puissent un jour concevoir Görlitz comme faisant partie intégrante de leurs loisirs.

s'agit d'un seul et même ensemble urbain. Des plans de la ville sur chacune des rives incluent d'ailleurs l'autre ville, montrant la partie allemande, plus importante au niveau du territoire et de la population ( 65000 habitants), reliée à la partie polonaise, plus petite ( 35000 habitants) par un pont unique. Cette agglomération d'environ 100000 habitants ne possède qu'une voie piétonnière pour traverser la rivière dont le niveau l'été ne dépasse guère la hauteur du genou. Le Pont de l'Amitié se trouve dans la partie sud de la ville, entre le quartier commerçant et la gare de Görlitz et le soi-disant nouveau quartier de Zgorzelec. 
Figure 2 : Plan des villes de Görlitz et de Zgorzelec

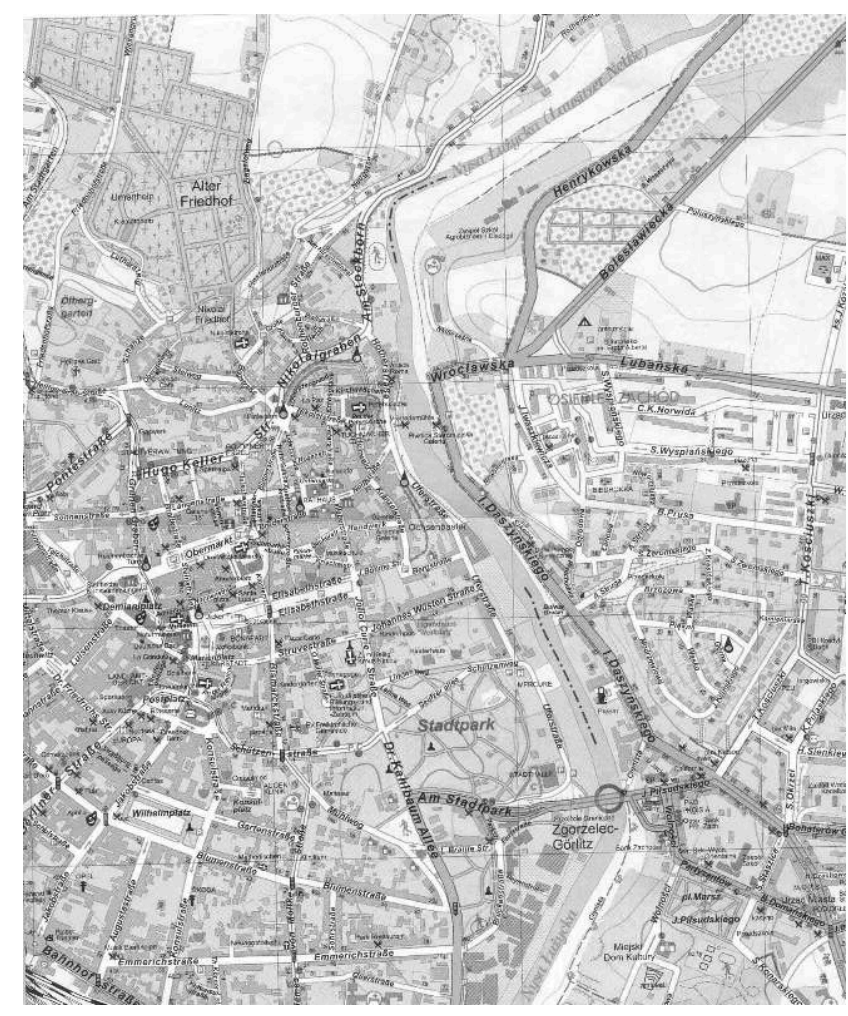

$33 \mathrm{Ne}$ serait-ce qu'en termes techniques, cela constitue un frein aux tentatives d'intégration des deux communautés. Au-delà de leurs différences de langue, de territoire, de nationalité et de culture, et du fait qu'elles n'ont pu que s'observer pendant un demi-siècle (les rapports personnels étant limités à un groupe de Polonais qui travaillaient dans les usines de Görlitz dans les années 70), ces deux communautés doivent, de surcroît, faire des efforts certains pour se rendre de l'autre côté de la rivière. Comme nous l'avons déjà mentionné ci-dessus, les autorités des deux villes espèrent reconstruire le pont de la vieille ville. Il relierait en fait deux restaurants, deux hostelleries emblématiques des deux communautés. L'idée de la reconstruction naquit il y a dix ans. Grâce au soutien des médias locaux, les deux communautés se sont ralliées au projet. Cela dit, bien que l'accord et le financement du projet soient acquis, la construction est retardée, apparemment par les gouvernements centraux. En effet, bien que ce pont constitue un moyen pratique de traverser la rivière pour les habitants de Görlitz et de Zgorzelec, c'est aussi un poste frontière international qui doit être reconnu par les autorités centrales ! En fait, la construction du pont entraîne la révision du traité bilatéral des frontières qui détermine le nombre de postes frontière entre les deux états.

Alors que la logique nous invite à voir le pont comme un moyen qui permettrait à des touristes, ayant essayé la bière de froment ou la «soljanka » à Görlitz, d'aller à Zgorzelec pour se régaler de 'knödel' ou de bière polonaise «Zywiec », les gouvernements centraux envisagent le pont en terme de financement de nouveaux postes de garde-frontière. Et pourtant, ce pont n'est pas attendu principalement par les retraités ouest-allemands en week-end à l'est, mais surtout par les quelques organisations qui tentent d'établir plus de contacts de part et d'autre de la frontière. Ce sont des personnes enthousiastes qui dépensent beaucoup de leur temps et, très 
souvent, de leur argent personnel pour rapprocher ces deux communautés et nations. Ils visent essentiellement les jeunes, les femmes, les retraités et les membres de l'église, mais la réponse des autres habitants de Zgorzelec reste très limitée. L'idée de ces personnes dynamiques cependant, c'est de générer des commentaires comme celui que l'on peut lire ci-dessous en traduction, exprimé par une femme qui ne semble pas remarquer la frontière, devenue trop banale :

Moi : Mais si vous voulez vous balader ou faire quelque chose de culturel...

M.F. : Alors tout le monde pense automatiquement à Görlitz.

Moi : Vraiment?

M.F. : Bien sûr.

Moi : Vous voulez dire vos amis?

M.F. : Oui, oui. Si, par exemple, je sais pas moi, quelqu'un venait me voir, comme un ami, ou me demandait où il peut se balader dans un joli coin, je dirais Görlitz et seulement Görlitz.

Pour cette jeune Polonaise, Görlitz, une ville dans un autre pays, séparée de Zgorzelec par la frontière de Schengen, est l'endroit où l'on va pour se balader. Cette affirmation qui place la ville allemande comme l'endroit où tout le monde va se balader est renforcée par des mots tels que na pewno (« automatiquement »), zdecydowanie (« bien sûr ») et tylko i wylacznie (" et seulement »). Il n'y a aucun doute que la ville au-delà du pont appartient à la vie et au paysage quotidien de cette jeune femme (sur la relation entre l'espace et l'identité, voir Buttimer et Seamons, 1980 ; Massey, 1994; Meegan, 1995 ; Galasinska et al.,2002).

Ceux qui veulent construire le pont, veulent voir des piétons tels que cette jeune fille l'emprunter. C'est d'ailleurs l'idée du pont qui inspira le restaurant et le café sur la rive polonaise, dans le vieux quartier de Zgorzelec qui n'est guère fréquenté par les visiteurs et plutôt laissé à l'abandon en attente d'une cure de jouvence. Ces établissements ont été montés par des entrepreneurs de Zgorzelec qui ont emprunté de l'argent et investi leurs propres fonds pour les ouvrir et qui, comme le pont ne se matérialise pas, craignent désormais pour leur avenir.

\section{En guise de conclusion}

Le commencement des travaux de construction du nouveau pont a été prévu pour le 28 avril 2003. Le 7 mai, jour du cinquième anniversaire de la création de l'Eurocité, la première pierre du pont a été posée, juste sous l'inauguration du monument WASE. La construction est financée par le côté allemand, en partie avec les fonds du programme de l'INTERREG III de l'Union européenne et en partie par le gouvernement de la Saxe. 


\section{BIBLIOGRAPHIE}

BACZYN'SKA ELZ.BIETA (1999). - Zjawiska patologii spoecznej w 'swiadomo'sci mieszka'nców pogranicza polsko niemieckiego. In Pogranicze z Niemcami a inne pogranicze Polski, edited by Zbigniew Kurcz. Wroclaw: Wydawnictwo Uniwersytetu Wroclawskiego, pp. 119140.

BUtTIMER A. and SEAMON D., eds. (1980).- The Human Experience of Space and Place. London: Croom Helm.

DonNAN H. \& WiLson T.M. (1994). - « An anthropology of frontiers ». In Donnan, H. \& Wilson T.M. (Eds.),Border approaches: anthropological perspectives on frontier (pp. 1-14). Lanham, MD: University Press of America.

DonNAN H. \& WiLSON T.M. (1999). - Borders. Frontiers of Identity, Nation and State. Oxford, New York: Berg.

EYLES John. (1985). - Sense of Place. Warrington: Silverbrook Press.

GALASI'NSKA A., ROLLO C, MEINHOF U.H. (2002). - « Urban Space and the Construction of Identity in a German-Polish Border ». InMEINHOF U.H. (ed.) Living (with) Borders. London: Ashgate.

GALASI'NSKI Dariusz and MEINHOF Ulrike H. (2002). - « Looking Across the River: German-Polish Border Communities and the Construction of the Other $»_{2}$ Journal of Language and Politics, 1 (1), pp. 23-58.

JAJESNIAK-QUAST D. \& STOKLOSA K. (2000). - Geteilte staedte an Oder und Neisse. Berlin Verlag.

KenNARD A. (2000). - « Transnational Planning in the German-Polish Border Region ». InAMSTRONG W., PARKER N. (eds.) Margins in European Integration. Macmillan.

KURZEPA Jacek (1999). - « Otwarcie granicy a nielegalna dziaalno's'c gospodarcza ». In Transgranicyno's' $c$ w perspektywie socjologicznej-kontynuacje, edited by Leszek Go $\geq$ dyka, Zielona Góra, 1999, pp. 255-270.

MASSEY Doreen (1994). - Space, Place and Gender. Cambridge: Polity Press.

MEEGAN Richard (1995). - « Local Worlds ». InGeographical Worlds, edited by John Allen and Doreen Massey.Oxford: OUP, pp. 53-104.

MEINHOF U.H. \& GALASI'NSKI D (2000). - «Photography, memory, and the construction of identities on the former East-West German border ».Discourse Studies, 2(3),pp. 323-53.

MEINHO U.H. \& GALASI'NSKI D. (2002). - « Reconfiguring Eas-West identities: cross-generational discourses in German and Polish border communities ».Journal of Ethnic and Migration Studies, 28 (1), pp. 63-82.

OseKowski Czesaw and Szczegóa Hieronim (1999). - Pogranicze Polsko-Niemieckie w okresie transformacji 1989-1997.Zielona Góra: WSP.

PEARSON Parker, RICHARDS Michael and Colin. (1994). - « Ordering the World: Perceptions of Architecture, Space and Time ». InArchitecture and Order. Approaches to Social Space, edited by Michael Parker Pearson and Colin Richards. London: Routledge, pp. 1-37.

RABINOWITZ D. (1994). - « To sell on not to sell? Theory versus practice, public versus private, and the failure of liberalism: the case of Israel and its Palestinian citizens ». American Ethnologist, 21 (4), pp. 827-844. 
SzLACHCICOWA Irena (1999). — « Toz samo's'c mieszka'nców polsko-niemieckiego pogranicza w Euroregionie Nysa ». InPogranicze z Niemcami a inne pogranicze Polski, edited by Zbigniew Kurcz. Wroclaw: Wydawnictwo Uniwersytetu Wroclawskiego, pp 71-82.

WILSON T.M. \& DONNAN H. (1998). - « Nation, state and identity at international borders ».InT.M. Wilson \& H. Donnan (Eds.) Border identities. Nation and state at international frontiers (pp. 1-30). Cambridge: Cambridge University Press.

\section{RÉSUMÉS}

Cet article examine les communautés de Görlitz et Zgorzelec, deux villes adjacentes au bord de la rivière Neisse/Nysa séparées par la frontière germano-polonaise. Les données sur lesquelles cette étude est basée sont issues d'un projet de recherche ethnographique qui couvre l'Europe entière et qui étudie les communautés vivant le long de la frontière entre l'Union européenne et ses pays limitrophes. Ce projet a été financé par le cinquième Programme Cadre de la Commission européenne. On examinera les deux niveaux de la coopération transfrontalière qui existe entre ces deux communautés, et que l'on peut métaphoriquement comparer aux deux niveaux du pont reliant les deux villes. Depuis la libéralisation du régime frontalier, il y a de cela plus d'une dizaine d'années, les deux municipalités ont établi une étroite collaboration qui a conduit à la création de l'« Euro-cité GR-ZG» en mai 1998. A l'image du pont, il y a un niveau supérieur et officiel représenté par les élites politiques, et le niveau inférieur, celui de la vie quotidienne des habitants de Görlitz et de Zgorzelec. Les résultats tirés des recherches effectuées sur le terrain montrent qu'il y a un hiatus et un manque de contact entre les deux niveaux. En d'autres termes, il existe un certain "décalage" entre les discours des décideurs politiques et la réalité des communautés locales. On examine également quelques problèmes démographiques, sociologiques et culturels auxquels chaque ville est confrontée et les solutions que leur coopération dans le cadre du projet « Euro-cité » peuvent y apporter.

The article focuses on the communities of Görlitz and Zgorzelec, two directly adjacent towns on the River Neisse/Nysa divided by the German-Polish border. The data comes from a Europe-wide ethnographic project studying communities living on the borders between the EU and its ascendant nations, funded by the European Commission's $5^{\text {th }}$ Framework Programme. I have a look at the two levels of the cross-border co-operation of the two communities, metaphorically rendered by the two levels of the bridge. Since the border regime was relaxed over a decade ago the two town authorities have co-operated very closely with one another, culminating in the establishment of the « Euro-town GR-ZG » in May 1998. Thus there is the upper and official level, represented by the political elites, and there is the lower level, one of the everyday life of the inhabitants of Görlitz and Zgorzelec. The results of the fieldwork carried out in the towns indicate a hiatus and a lack of contact between the two levels, or, to put it differently, between the discourses of the policy makers and local communities. I describe some of the demographic, sociological and cultural problems each town is facing and some of the ways in which their cooperation under the 'Euro-town' umbrella can provide solutions to these problems.

Dieser Beitrag stellt die beiden Gemeinden Görlitz und Zgorzelec vor, zwei direkt benachbarte Städte an der Neisse/Nysa, getrennt durch die deutsch-polnische Grenze. Die empirischen Daten wurden in einem ethnographischen europäischen Projekt erhoben, das von der Europäischen Kommission im 5.Rahmenprogramm finanziert wurde. In diesem Projekt wurden Gemeinden an der EU-Aussengrenze und ihre Nachbargemeinden in den östlichen Nachbarstaaten untersucht. In diesem Artikel werden zwei Ebenen der grenzüberschreitenden Zusammenarbeit der beiden 
Städte metaphorisch verknüpft mit den zwei Ebenen der Brücke, welche die beiden Städte verbindet. Seit die Grenzbewachung vor 10 Jahren reduziert wurde, arbeiten die beiden Stadtregierungen eng zusammen, wobei der Höhepunkt mit der Einrichtung der « Euro-Stadt GRZG» im Mai 1998 erreicht wurde. Dies alles läuft auf der oberen, der offiziellen Ebene, die von den politischen Eliten repräsentiert wird, aber es gibt auch die quasi untere Ebene, die des täglichen Lebens der Einwohner und Einwohnerinnen von Görlitz und Zgorzelec. Die Resultate der Feldarbeit in den beiden Städten zeigen eine Bruchlinie und einen Mangel an Übereinstimmung zwischen den beiden Ebenen, oder, um es anders zu sagen, zwischen den Diskursen der Politiker und der lokalen Gesellschaft. Darüber hinaus werden einige der demographischen, sozialen und kulturellen Probleme, mit denen die beiden Städte konfrontiert sind, beschrieben sowie die Möglichkeiten, wie diese Probleme durch die Zusammenarbeit als Euro-Stadt gelöst werden könnten.

INDEX

Mots-clés : Allemagne, coopération transfrontalière, Euro-cité, Pologne, villes frontalières

Keywords : border communities, cross-border co-operation, Euro-town, Germany, Poland

Schlüsselwörter : Deutschland, Euro-Stadt, Grenzstädte, grenzüberschreitende Zusammenarbeit, Polen

\section{AUTEURS}

\section{ALEKSANDRA GALASINSKA}

School of Humanities, Languages and Social Sciences - University of Wolverhampton - Wulfruna Street, Wolverhampton WV1 1SB, UK - a.galasinska@wlv.ac.uk 